\title{
Caulophyllum thalictroides Root
}

National Cancer Institute

\section{Source}

National Cancer Institute. Caulophyllum thalictroides Root. NCI Thesaurus. Code C72494.

A herbal remedy derived from the root of the perennial flowering plant Caulophyllum thalictroides grown in the eastern North America. Blue cohosh root contains a variety of compounds, including the alkaloid N-methylcytisine and the saponin caulosaponin. Blue Cohosh is typically in the form of a tincture or a decoction. 